\title{
Standardized method for the measurement of Grabb's line and clival-canal angle
}

\author{
Jonathan E. Martin, MD, ${ }^{1}$ Markus Bookland, MD, ${ }^{1}$ Douglas Moote, MD, ${ }^{2}$ and Catherine Cebulla ${ }^{1}$ \\ 1Division of Neurosurgery, Department of Surgery, Connecticut Children's Medical Center; and 2Department of Radiology, \\ Connecticut Children's Medical Center, Hartford, Connecticut
}

\begin{abstract}
OBJECTIVE Grabb's line-the perpendicular distance from the basion-C2 line (pB-C2) - is a widely used radiographic measurement with significant clinical implications in patients with a complex Chiari malformation. Rigorous demonstration of the reproducibility of this measurement has not previously been reported. The authors report a standardized measurement technique with excellent inter- and intrarater reliability on T1-weighted sagittal MRI.

METHODS The authors developed a standardized measurement technique that included specifications of midline slice selection, landmark and reference line definitions, and measurement technique on T1-weighted sagittal images. Twenty MR images were reviewed by 2 pediatric neurosurgeons, 1 pediatric radiologist, and 1 undergraduate student. Measurements were performed using the technique specified on 2 separate occasions. Intrarater and interrater reliabilities were calculated using the intraclass correlation coefficient.

RESULTS A combined interrater reliability of 0.879 was achieved for the pB-C2, and 0.916 for the clival-canal angle, another measure of interest in patients with complex Chiari malformations. Intrarater reliability for these measurements exceeded 0.858 for all 4 reviewers.

CONCLUSIONS Grabb's line-the pB-C2_can be measured with excellent reliability using a standardized measurement protocol. Individual clinicians and collaborative databases should consider using validated measurement techniques to guide clinical decision making in patients with craniocervical junction pathology.

https://thejns.org/doi/abs/10.3171/2017.5.PEDS17181
\end{abstract}

KEY WORDS Grabb's line; pB-C2; Chiari l; ventral brainstem compression; diagnostic technique

$\mathrm{M}$ EASUREMENT precision is critical to the establishment of a clinically useful radiographic measurement. Since its initial description in 1998, Grabb's line-the perpendicular distance from the basion$\mathrm{C} 2$ line (pB-C2) - has been adopted by many in the neurosurgical community as a quantifiable measure of ventral brainstem compression. ${ }^{5}$ In combination with the work of Menezes, ${ }^{8,9}$ it has been used to justify occipital-cervical fusion in selected patients. ${ }^{1}$ Although generally regarded as easily reproducible, ${ }^{2}$ recent attempts to rigorously assess the interrater reliability of Grabb's line have shown poor inter- and intrarater reliability ${ }^{1}$ (see also Uribe R, Huang K, Souweidane M: "Intra and interobserver reliability of the pB-C2 line in patients with Chiari malformation"-poster presented at the 45th Annual Section on Pediatric Neurological Surgery, Orlando, FL, December 2016). Given the reduction in cervical mobility and increased potential for morbidity in a fusion procedure relative to a more standard suboccipital decompression, confidence in this measure- ment technique would seem to be of critical importance to neurosurgeons and orthopedic surgeons with an interest in craniocervical pathology.

\section{Methods}

Twenty consecutive MRI scans from our facility (Siemens 1.5-T unit) were reviewed by 2 attending pediatric neurosurgeons, 1 pediatric radiologist, and 1 undergraduate student. Brain, cervical spine, and cervicothoracic spine studies were included to replicate the imaging commonly available at the time of patient referral. To limit confounding anatomical variation and imaging artifacts, patients with segmentation anomalies or orthodontic hardware were excluded.

A 6-step measurement technique guide was provided to reviewers for the completion of all measurements (see Fig. 4 and Table 1). Full instructions and reference figures can be obtained at https://connecticutchildrens.org/search-spe cialties/neurosurgery/neurosurgery-resources/. A synopsis

ABBREVIATIONS CP = clival point; CXA = clival-canal angle; ICC = intraclass correlation coefficient; $\mathrm{pB}-\mathrm{C} 2$ = perpendicular distance from the basion-C2 line; $\mathrm{SS}=$ slice selection.

SUBMITTED April 9, 2017. ACCEPTED May 10, 2017.

INCLUDE WHEN CITING Published online August 11, 2017; DOI: 10.3171/2017.5.PEDS17181. 


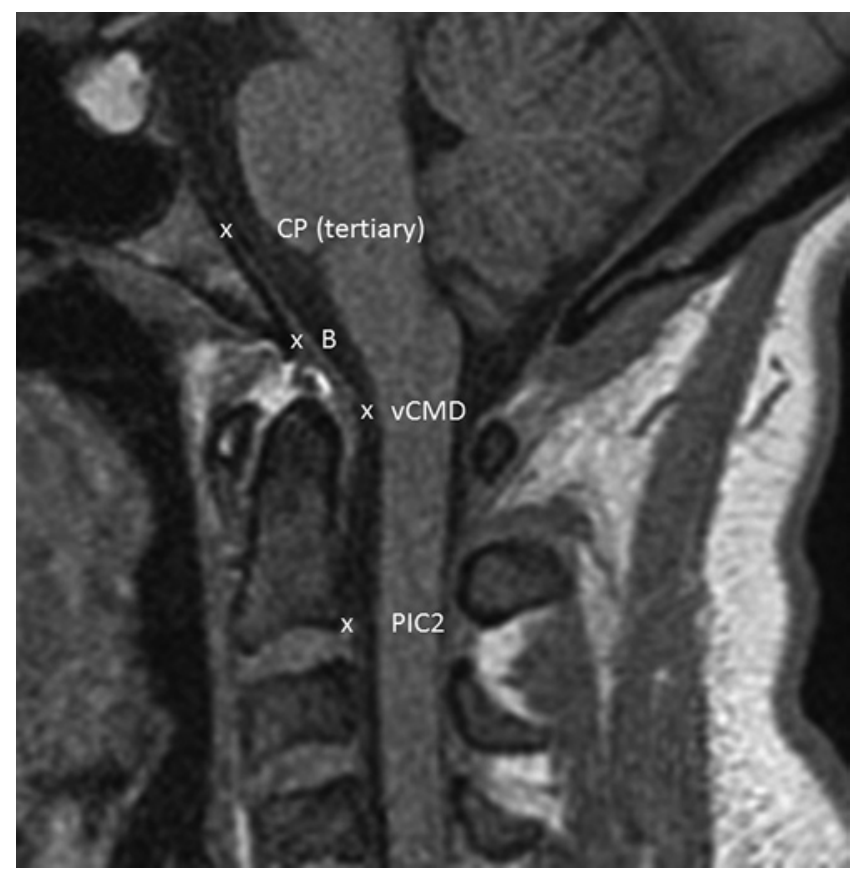

FIG. 1. Instructions to reviewers for the determination of radiographic landmarks used as the basis for measurement on T1-weighted sagittal imaging. The CP is the point of intersection between the clival surface (represented by the T1-weighted stripe of cortical bone) and 1 of 3 prioritized landmarks based on bone age of the patient and field of view of the study (the tertiary definition is used in this figure). The basion (B) is the most posterior point of extension of the clivus. The ventral cervicomedullary dura (vCMD) is the most dorsal point on the ventral dural margin at the cervicomedullary junction. The posteroinferior cortex of $\mathrm{C}-2$ (PIC2) is the most dorsal point of the inferior end plate of C-2.

of these instructions is provided as follows. Reviewers first identified the midline image used for all measurements. The T1 sagittal image with the most superior extension of the odontoid (or C2 body if odontoid poorly visualized) was selected. Exception to this rule was granted in the setting of a bifid odontoid tip with midline saddle, with the reviewer independently determining the most midline slice.
Following this, 3 bony and 1 soft-tissue anatomic landmarks were precisely defined (Figs. 1 and 2). The definition of the clival point (CP) deserves specific mention. Based on the variability of clival anatomy due to the imaging field of view and patient bone age, a 3-tiered definition of the clival point was developed to allow for clear definition on all patients and radiographs (Fig. 2). Reference lines allowing for the measurement of Grabb's line and clival canal angle (CXA), another frequently employed measure of craniocervical junction morphology in patients with Chiari malformation, ${ }^{1}$ were then drawn (Fig. 3). An illustration of the clinical measurements is presented in Fig. 4.

Images were reviewed on diagnostic-quality 5-megapixel monitors (Barco), using our institutional PACS viewer (Phillips iSite). Monitor settings included default modality settings and a zoom preset of $400 \%$. Measurements were performed twice by each reviewer to an accuracy of $0.1 \mathrm{~mm}$. To eliminate measurement bias related to provider recall or proficiency, reviewers were instructed to complete measurements with a minimum of 1 week between sessions, and the image list was reordered for each measurement session.

Descriptive statistics were calculated for the continuous variables of the $\mathrm{pB}-\mathrm{C} 2$ and $\mathrm{CXA}$. The intraclass correlation coefficient (ICC) $)^{11}$ was calculated on the assumption that the observers represented a random sample of the population, to permit generalizability of the findings of the study. Single rather than mean measurements were considered for the purposes of analysis. The relative fidelity of reliability measurements was judged in accordance with the following established ranges: poor, $0-0.5$; moderate, 0.5-0.75; good, $>0.75 .^{10}$

\section{Results}

Twenty consecutive MR images meeting inclusion criteria were used in this analysis. Specific imaging studies included $17 \mathrm{MR}$ images of the brain, $1 \mathrm{MR}$ image of the cervical spine, and $2 \mathrm{MR}$ images of the cervicothoracic spine. The mean age at the time of the studies was 10.4 years (range 6 months-19 years), and the proportion be-
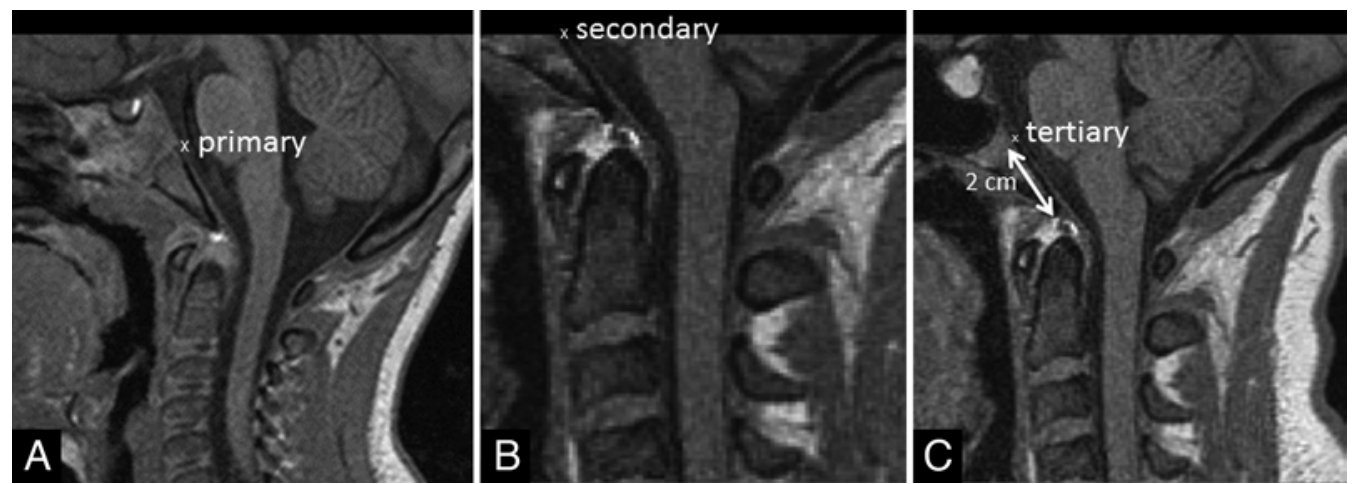

FIG. 2. A 3-tiered definition of the CP based on bone age and imaging field of view. A: Primary definition: the most dorsal extension of the cortical bone of the clivus (low-signal T1-weighted stripe) at the superodorsal margin of the sphenooccipital suture. B: Secondary definition: the most dorsal extension of the cortical bone of the clivus (low T1-weighted stripe) at the highest visible point included within the field of view. This definition is used when the superodorsal margin of the sphenooccipital suture is not visible within the field of view. C: Tertiary definition: the most dorsal extension of the cortical bone of the clivus (low T1-weighted stripe) at a point 2 linear centimeters above the basion along the clival surface. This definition is used when the sphenooccipital suture is not visible due to advanced bone age. 


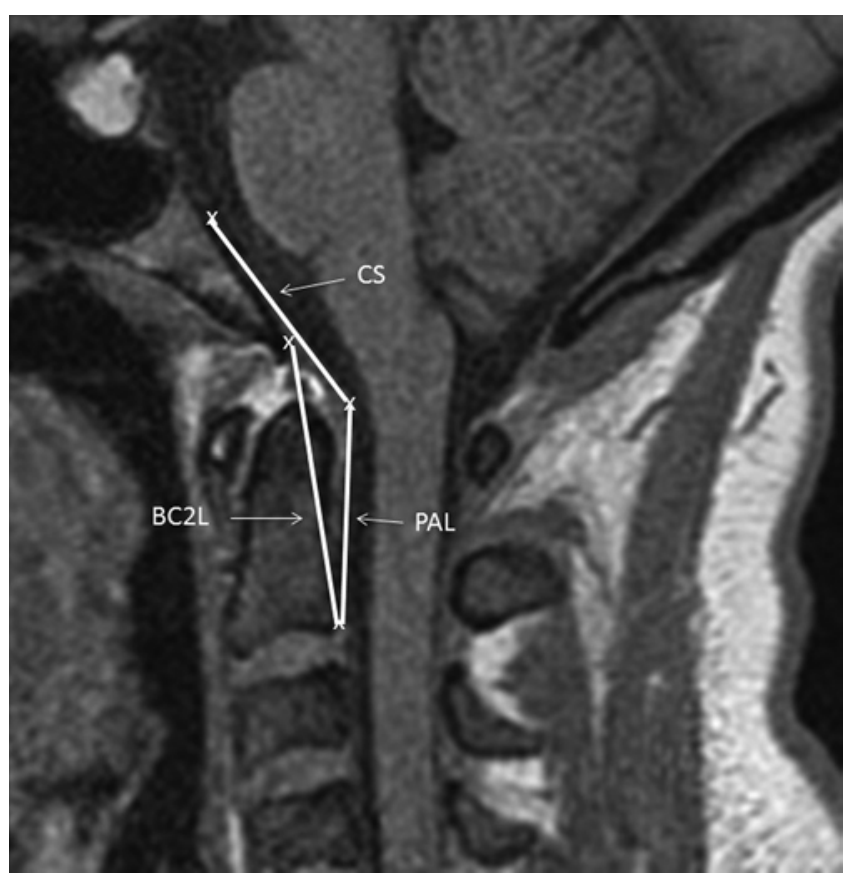

FIG. 3. Instructions to reviewers for creation of reference lines used for the measurement of Grabb's line and the CXA. The clival slope (CS) connects $C P$ to $v C M D$. The posterior axial line (PAL) connects VCMD to the posteroinferior cortex of $\mathrm{C}-2$. The basion- $\mathrm{C} 2$ line $(\mathrm{BC} 2 \mathrm{~L})$ connects basion to the posteroinferior cortex of $\mathrm{C}-2$.

tween the sexes (9 male and 11 female patients) was nearly equal.

Midline slice selection (SS) was recorded by reviewers and was highly reproducible, with an interrater reliability ICC of 0.999. The Grabb's line measurements ranged from 2.3 to $10.3 \mathrm{~mm}$, with a mean of $6.5 \mathrm{~mm}$. The CXA measurements ranged from $120^{\circ}$ to $170^{\circ}$, with a mean of $144.5^{\circ}$.

Overall measurement fidelity, as assessed by ICC for inter- and intraobserver analysis, is presented in Tables 2 and 3. Overall interrater reliability for the $\mathrm{pB}-\mathrm{C} 2$ was 0.879 (95\% CI 0.797-0.942) and for the CXA it was 0.916 (95\% CI 0.854-0.960). Intrarater ICCs for both the pB-C2 and CXA were exceptional for all reviewers (Fig. 5). The ICC ranged from 0.858 to 0.963 for the $\mathrm{pB}-\mathrm{C} 2$ and 0.859 to 0.982 for the CXA. No correlation between years of clinical experience and measurement fidelity was apparent.

\section{Discussion}

The reliability of radiographic measurements of the craniocervical junction has historically been poor. ${ }^{6,12,13}$ Factors cited in low measurement fidelity include image quality, variable landmark identification, and the skill level of the observers. ${ }^{13}$ Improvements in measurement fidelity have been noted with the advent of digital radiography. ${ }^{3}$ Although digital technology has clear advantages over manual measurement techniques, it does nothing to address factors such as landmark identification or techniques for drawing reference lines for measurement. ${ }^{4}$

Grabb's line was the first linear, quantifiable measurement of ventral brainstem compression. It has subsequently been applied to guide selection of the surgical approach in patients with Chiari malformation. ${ }^{1,7}$ Some authors have justified primary occipital-cervical fusion for patients with a pB-C2 exceeding $9 \mathrm{~mm}$ (see Loven T, White T, Nguyen V, Aygok G, Bolognese P, Kula R, Rekate HL: "Assessment of anterior pathology in symptomatic patients with hindbrain herniation [Chiari 1 malformation];" poster presented at the 43rd Annual Section on Pediatric Neurological Surgery, Amelia Island, FL, December
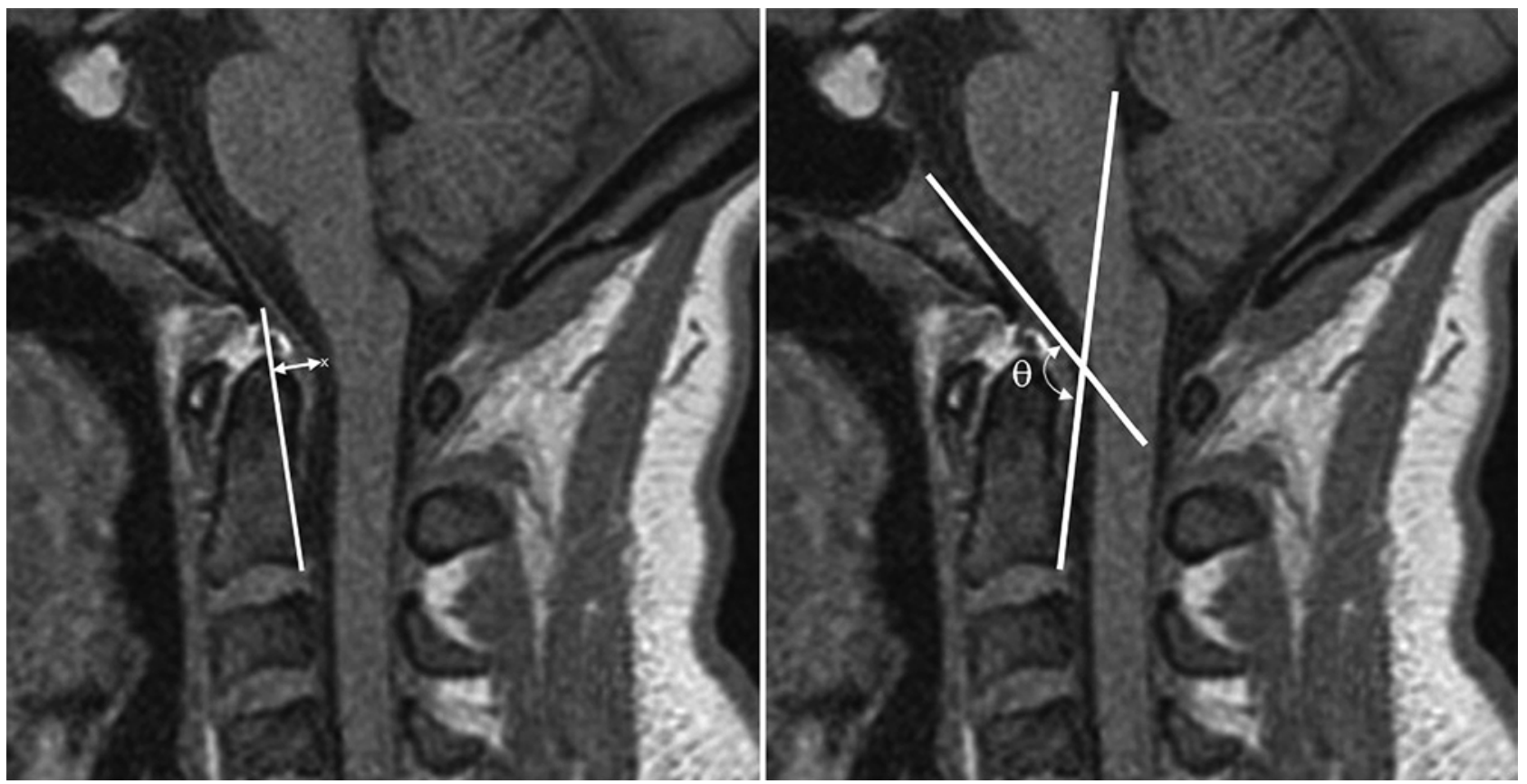

FIG. 4. Illustrations of clinical measurements. Left: Grabb's line (pB-C2). Right: The CXA ( $\Theta)$. 
TABLE 1. Measurement technique

\begin{tabular}{|c|c|}
\hline Step & Description \\
\hline 1 & Confirm appropriate windowing \& magnification \\
\hline 2 & $\begin{array}{l}\text { Identify midline slice: identify slice w/ the most superior } \\
\text { extension of the odontoid (or C-2 body if odontoid is } \\
\text { poorly visualized). In the case of a bifid odontoid tip w/ } \\
\text { a midline "saddle," select the most midline slice relative } \\
\text { to the clivus \& C-2/odontoid. }\end{array}$ \\
\hline \multirow[t]{7}{*}{3} & Identify key landmarks (Figs. $1 \&$ 2) \\
\hline & $\begin{array}{l}\text { Bony landmarks: use modality default settings w/ no } \\
\text { image processing }\end{array}$ \\
\hline & $\mathrm{CP}$ \\
\hline & B \\
\hline & PIC2 \\
\hline & $\begin{array}{l}\text { Soft-tissue landmarks: use post-processing image in- } \\
\text { version to improve visualization }\end{array}$ \\
\hline & vCMD \\
\hline \multirow[t]{4}{*}{4} & Construct requisite reference lines (Fig. 3) \\
\hline & BC2L: connect B to PIC2 \\
\hline & CS: connect CP to vCMD \\
\hline & PAL: connect PIC2 to vCMD \\
\hline \multirow[t]{4}{*}{5} & $\begin{array}{l}\text { Perform measurement of perpendicular distance btwn } \\
\text { vCMD \& BC2L (Fig. 4) }\end{array}$ \\
\hline & $\begin{array}{l}\text { Rotate image } 90^{\circ} \text { to place } \mathrm{BC} 2 \mathrm{~L} \text { in near-horizontal } \\
\text { plane w/vCMD above the line }\end{array}$ \\
\hline & $\begin{array}{l}\text { Apply post-processing image inversion to select vCMD, } \\
\text { adjust CS \& PAL accordingly }\end{array}$ \\
\hline & $\begin{array}{l}\text { Measure perpendicular distance btwn vCMD \& BC2L, } \\
\text { \& record as pB-C2 }\end{array}$ \\
\hline 6 & Perform measurement of CXA (Fig. 4) \\
\hline & Perform angular measurement btwn CS \& PAL \\
\hline
\end{tabular}

$\mathrm{B}=$ basion; $\mathrm{BC} 2 \mathrm{~L}=$ basion $-\mathrm{C} 2$ line; $\mathrm{CS}=$ clival slope; $\mathrm{PAL}=$ posterior axial line; $\mathrm{PIC2}=$ posterior-inferior cortex of $\mathrm{C}-2 ; \mathrm{vCMD}=$ ventral cervicomedullary dura.

2014). Given the previously discussed challenges with radiographic assessment of the craniocervical junction, use of Grabb's line for such purposes seems problematic when the reliability and reproducibility of this measurement has not been established.

We determined a set of principles that we thought were critical to our success in achieving a useful and adequately precise measurement technique. These were as follows: 1) T1-weighted sagittal images were selected for use, based on their universal availability on both brain and cervical spine MRI. 2) A technique was specified for the selection of the midline slice to be used for measurement. 3) Precise text and pictorial descriptions of landmarks, refer-

TABLE 2. Interrater reliability of SS, pB-C2, and CXA

\begin{tabular}{cccc}
\hline Measurement & ICC & $95 \% \mathrm{Cl}$ & $\mathrm{p}$ Value \\
\hline $\mathrm{SS}$ & 0.999 & $0.998-0.999$ & $<0.05$ \\
\hline $\mathrm{pB}-\mathrm{C} 2$ & 0.879 & $0.797-0.942$ & $<0.05$ \\
\hline CXA & 0.916 & $0.854-0.960$ & $<0.05$ \\
\hline
\end{tabular}

TABLE 3. Intrarater reliability of SS, pB-C2, and CXA for Observers 1-4, in ascending order of clinical experience

\begin{tabular}{|c|c|c|c|c|}
\hline Observer No. & Measurement & ICC & $95 \% \mathrm{Cl}$ & p Value \\
\hline \multirow{3}{*}{$\begin{array}{l}\text { 1; undergraduate } \\
\text { student }\end{array}$} & SS & 0.997 & $0.993-0.999$ & $<0.05$ \\
\hline & $\mathrm{pB}-\mathrm{C} 2$ & 0.885 & $0.732-0.953$ & $<0.05$ \\
\hline & CXA & 0.921 & $0.811-0.968$ & $<0.05$ \\
\hline \multirow{3}{*}{$\begin{array}{c}\text { 2; PGY 11, ped } \\
\text { neurosurg }\end{array}$} & SS & 0.999 & $0.999-1.000$ & $<0.05$ \\
\hline & $\mathrm{pB}-\mathrm{C} 2$ & 0.919 & $0.807-0.967$ & $<0.05$ \\
\hline & CXA & 0.892 & $0.748-0.956$ & $<0.05$ \\
\hline \multirow{3}{*}{$\begin{array}{c}\text { 3; PGY 20, ped } \\
\text { neurosurg }\end{array}$} & SS & 0.999 & $0.998-1.000$ & $<0.05$ \\
\hline & $\mathrm{pB}-\mathrm{C} 2$ & 0.858 & $0.676-0.942$ & $<0.05$ \\
\hline & CXA & 0.859 & $0.678-0.942$ & $<0.05$ \\
\hline \multirow[t]{3}{*}{ 4; PGY 34, ped rad } & SS & 1.000 & $1.000-1.000$ & $<0.05$ \\
\hline & $\mathrm{pB}-\mathrm{C} 2$ & 0.963 & $0.908-0.985$ & $<0.05$ \\
\hline & CXA & 0.982 & $0.956-0.993$ & $<0.05$ \\
\hline
\end{tabular}

Ped neurosurg = pediatric neurosurgeon; ped rad = pediatric radiologist; $P G Y$ $=$ postgraduate year.

ence lines, and measurement techniques were drafted and used by reviewing clinicians at the time the measurements were performed. 4) Monitor settings were standardized and used by all reviewers.

Other authors have noted improved fidelity of measurement using T2-weighted sagittal sequences. ${ }^{2}$ Whereas this sequence is commonly available on cervical spine MRI, it is rarely obtained as a portion of an MR image of the brain. In contrast, T1-weighted sagittal imaging is routinely found on both of these studies. Our desire for a widely applicable measurement standard led us to choose the T1weighted sagittal sequence for this study.

We found identification of the ventral cervicomedullary dura margin to be excellent when we used postprocessing image inversion such as the "edge detect" feature available with our PACS viewer. Image inversion is a nearly universal feature present on radiology viewers. Use of this means of image manipulation provides a similar contrast between the CSF and soft-tissue signal to that seen on T2-weighted imaging. During the development of our measurement technique, we observed improved fidelity by converting the $\mathrm{pB}-\mathrm{C} 2$ measurement from a longitudinal measurement (side-to-side) to a latitudinal measurement (up/down). The necessity of image inversion and rotation can be debated. Future directions for study include an external validation of our methodology. Given the clinical significance assigned to these measurements, the fidelity demonstrated in this study should prompt strong consideration of adherence to the described technique.

\section{Conclusions}

We have demonstrated a proof of concept that Grabb's line can be quantified with excellent inter- and intraobserver reliability by using a rigidly defined technique. The technique described in this paper applies only to T1-weighted sagittal MRI; it cannot be assumed that this 


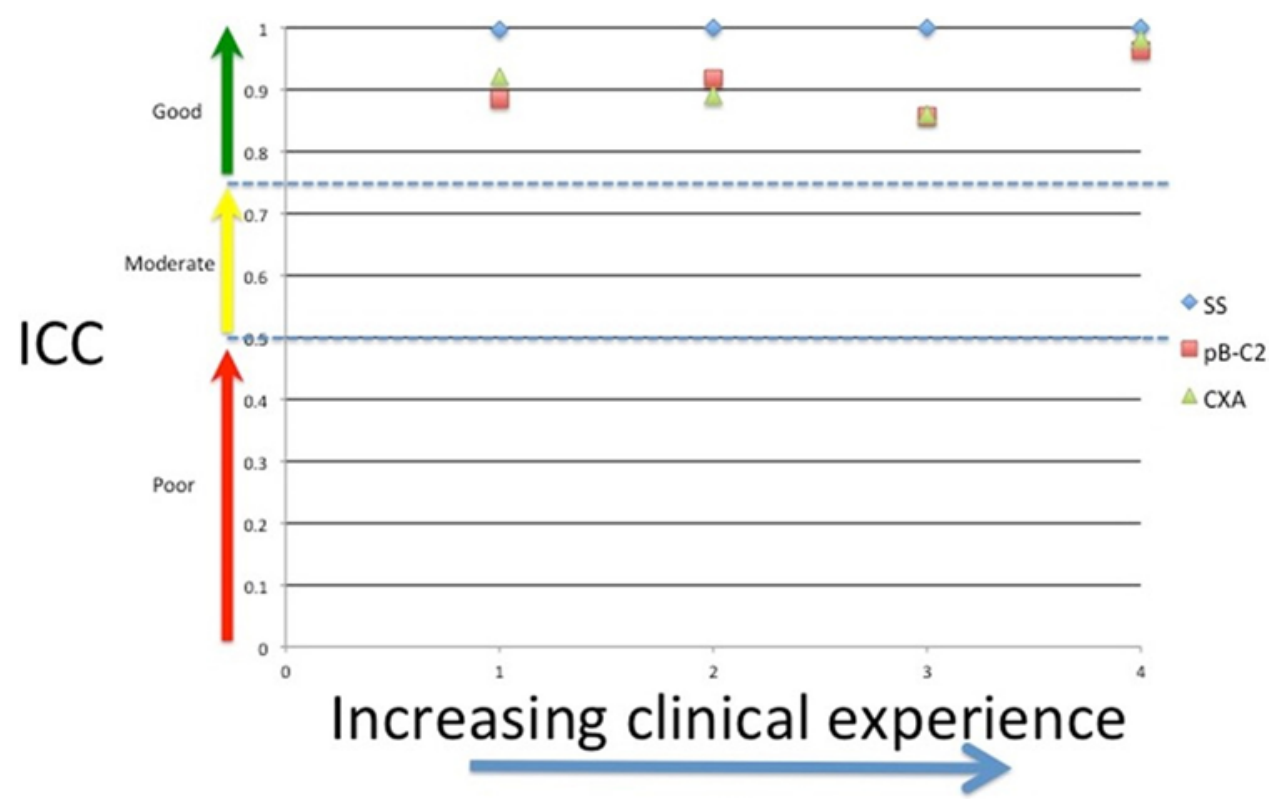

FIG. 5. Graph illustrating the data in Table 3: the intrarater reliability for SS, pB-C2, and CXA. All measurements exceeded the threshold of 0.75 for ICC required for assignment of "good" fidelity. Note the lack of correlation between clinical experience and measurement fidelity. Confidence intervals are included. Figure is available in color online only.

technique is valid for CT images of the craniocervical junction. Measurement fidelity was shown to be excellent regardless of clinical experience or specialty training in radiology or pediatric neurosurgery. Individual clinicians and collaborative databases should consider using validated measurement techniques to guide clinical decision making in patients with craniocervical junction pathology that includes complex Chiari malformation.

\section{Acknowledgments}

We thank members of the Pediatric Craniocervical Society for their encouragement of this project.

\section{References}

1. Bollo RJ, Riva-Cambrin J, Brockmeyer MM, Brockmeyer DL: Complex Chiari malformations in children: an analysis of preoperative risk factors for occipitocervical fusion. $\mathbf{J}$ Neurosurg Pediatr 10:134-141, 2012

2. Bonney PA, Maurer AJ, Cheema AA, Duong Q, Glenn CA, Safavi-Abbasi S, et al: Clinical significance of changes in pB-C2 distance in patients with Chiari Type I malformations following posterior fossa decompression: a single-institution experience. J Neurosurg Pediatr 17:336-342, 2016

3. Douglas TS, Sanders V, Machers S, Pitcher R, van As AB: Digital radiographic measurement of the atlantodental interval in children. J Pediatr Orthop 27:23-26, 2007

4. Gordon JE, Chen RC, Dobbs MB, Luhmann SJ, Rich MM, Schoenecker PL: Interobserver and intraobserver reliability in the evaluation of mechanical axis deviation. J Pediatr Orthop 29:281-284, 2009

5. Grabb PA, Mapstone TB, Oakes WJ: Ventral brain stem compression in pediatric and young adult patients with Chiari I malformations. Neurosurgery 44:520-528, 1999

6. Hinck VC, Hopkins CE: Measurement of the atlanto-dental interval in the adult. Am J Roentgenol Radium Ther Nucl Med 84:945-951, 1960

7. Ladner TR, Dewan MC, Day MA, Shannon CN, Tomycz L, Tulipan N, et al: Evaluating the relationship of the $\mathrm{pB}-\mathrm{C} 2$ line to clinical outcomes in a 15-year single-center cohort of pediatric Chiari I malformation. J Neurosurg Pediatr 15:178-188, 2015

8. Menezes AH: Decision making for management of craniovertebral junction pathology. Oper Tech Neurosurg 8:125130,2005

9. Menezes AH, VanGilder JC: Transoral-transpharyngeal approach to the anterior craniocervical junction. Ten-year experience with 72 patients. J Neurosurg 69:895-903, 1988

10. Portney LG, Watkins MP: Foundations of Clinical Research: Applications to Practice. Philadelphia: F. A. Davis Company, 2015, p 82

11. Shrout PE, Fleiss JL: Intraclass correlations: uses in assessing rater reliability. Psychol Bull 86:420-428, 1979

12. Smoker WRK: Craniovertebral junction: normal anatomy, craniometry, and congenital anomalies. Radiographics 14:255-277, 1994

13. Wellborn CC, Sturm PF, Hatch RS, Bomze SR, Jablonski K: Intraobserver reproducibility and interobserver reliability of cervical spine measurements. J Pediatr Orthop 20:66-70, 2000

\section{Disclosures}

The authors report no conflict of interest concerning the materials or methods used in this study or the findings specified in this paper.

\section{Author Contributions}

Conception and design: Martin. Acquisition of data: all authors. Analysis and interpretation of data: Martin, Bookland. Drafting the article: Martin. Critically revising the article: all authors. Reviewed submitted version of manuscript: Martin. Approved the final version of the manuscript on behalf of all authors: Martin. Statistical analysis: Martin.

\section{Correspondence}

Jonathan E. Martin, Department of Surgery, Division of Neurosurgery, University of Connecticut School of Medicine, Connecticut Children's Medical Center, 282 Washington St., Hartford, CT 06106.email: jmartin03@ccmckids.org. 